\title{
Surgical outcomes of Ahmed glaucoma valve implantation in patients with glaucoma secondary to iridocorneal endothelial syndrome
}

\author{
Zhen $\mathrm{Mao}^{1} \cdot$ Xinxing Guo ${ }^{2} \cdot$ Yimin Zhong $^{1} \cdot$ Xing Liu $^{1}$
}

Received: 9 June 2019 / Revised: 15 April 2020 / Accepted: 17 April 2020 / Published online: 4 May 2020

(c) The Author(s), under exclusive licence to The Royal College of Ophthalmologists 2020

\begin{abstract}
Objectives To evaluate the long-term outcome of patients with iridocorneal endothelial (ICE) syndrome who underwent Ahmed glaucoma valve implantation surgery for uncontrolled glaucoma.

Methods Eighteen patients who suffered from unilateral ICE syndrome with uncontrolled glaucoma and subsequently underwent Ahmed aqueous shunt surgery at Zhongshan Ophthalmic Center between January 2008 and December 2016 were reviewed. Outcome measures included intraocular pressure (IOP), the use of glaucoma medications, visual acuity, further surgical interventions, and surgical complications.

Results The mean IOP was reduced from $34.8 \pm 10.6 \mathrm{mmHg}$ on $3.6 \pm 0.5$ medications to $17.4 \pm 4.9 \mathrm{mmHg}(t=6.791, P=$ $0.000)$ on $1.6 \pm 1.1$ medications $(Z=-3.545, P=0.000)$ at the last follow-up $(42.0 \pm 19.3$ months). Five eyes $(27.8 \%)$ achieved complete success, nine $(50.0 \%)$ achieved qualified success, and the remaining four $(22.2 \%)$ were considered failures. Survival was $94.4 \%$ at 1 year, $88.1 \%$ at 2 years, and $73.5 \%$ at 3 years. Four cases displayed a flat anterior chamber and were treated with a single anterior chamber reformation surgery with no recurrence. No other complications related to the glaucoma drainage implants occurred in this series.

Conclusions Ahmed glaucoma valve implantation appears to be a safe and effective method for treating glaucoma secondary to ICE syndrome. Postoperative shallow anterior chamber and hypotony may occur but responds well to the treatment. Early consideration may be given to aqueous shunt surgery in patients with glaucoma secondary to ICE syndrome when trabeculectomy fails.
\end{abstract}

\section{Introduction}

Iridocorneal endothelial (ICE) syndrome is a rare disease characterized by corneal endothelial abnormality, peripheral anterior synechiae (PAS), iris stromal abnormalities, and secondary glaucoma. This syndrome can be subdivided into progressive iris atrophy, Chandler's syndrome, and CoganReese syndrome based primarily on changes in the iris. Chronic corneal edema results from the decrease in endothelial density as the corneal endothelial cells migrate to the

Xing Liu

drliuxing@163.com

1 State Key Laboratory of Ophthalmology, Zhongshan Ophthalmic Center, Sun Yat-Sen University, Guangzhou, PR China

2 Dana Center for Preventive Ophthalmology at the Johns Hopkins Wilmer Eye Institute, Baltimore, MD, USA periphery and are no longer confined to the posterior corneal surface by "contact inhibition". Abnormal endothelial cells also migrate over the trabecular meshwork and anterior portion of the iris, resulting in PAS, progressive closure of the anterior chamber angle, obstruction of the trabecular meshwork, and an intractable elevation of intraocular pressure (IOP) [1-3].

The prevalence of secondary glaucoma associated with ICE syndrome has been reported to range from 46 to $82 \%$, and glaucoma is the main reason for vision loss in patients with ICE syndrome [4-6]. In most cases, the glaucoma is refractory. Medical treatment usually tends to be ineffective. Filtering surgery often succeeds for a short time but frequently fails, presumably because of continued growth of the endothelial membrane over the surgical fistula [5, 7-10]. Laganowski et al. [5] demonstrated 60\% survival at 1 year and $21 \%$ survival at 5 years after the first trabeculectomy, and the survival fell to $20 \%$ at 1 year after the second trabeculectomy in a series of 22 patients. In a series of 15 
ICE patients, Chandran et al. [10] reported moderate surgical success with mitomycin-c (MMC) augmented trabeculectomy, with a complete success rate of $64 \%$ at 12 months, $57 \%$ at 36 months, and $33 \%$ at 60 months.

Doe et al. [8] reported survival rates of $73 \%$ at 1 year, $44 \%$ at 3 years, and only $29 \%$ at 5 years in twelve ICE patients undergoing trabeculectomy with antifibroblastic adjuncts. Meanwhile, 21 eyes in the series receiving aqueous shunt implantation achieved survival rates of $71 \%$ at 1 year, $71 \%$ at 3 years and $53 \%$ at 5 years. Aqueous shunt implantation is appealing to patients with ICE syndrome because it may bypass the chief hazard of filtration procedures: the regrowth of an ICE membrane over the ostium. However, few previous studies have focused on the efficacy of glaucoma drainage devices in controlling glaucoma in ICE syndrome. The purpose of the current study was to analyze the long-term outcome of patients who underwent aqueous shunt surgery for glaucoma secondary to ICE syndrome.

\section{Methods}

\section{Patient eligibility and recruitment}

Eighteen eyes of 18 patients who suffered from ICE syndrome with uncontrolled IOP and subsequently underwent aqueous shunt surgery at Zhongshan Ophthalmic Center between January 2008 and December 2016 were enrolled in this study. Uncontrolled IOP was defined as IOP $>21$ $\mathrm{mmHg}$ on fully tolerated topical medications. Ten of the eighteen eyes had undergone previous trabeculectomy and were classified as the "Trab group", six of which were supplemented with MMC. The other eight patients who had not undergone any prior glaucoma filtering surgeries were included in the "no-Trab group".

The study adhered to the tenets of the Declaration of Helsinki and was approved by the institutional review board and the ethics committee of Zhongshan Ophthalmic Center. The nature of the study and its possible consequences were explained to all participants, and written informed consent was obtained.

\section{Surgical procedures}

All aqueous shunt implantation surgeries were performed by the same surgeon (Professor Liu) using similar surgical techniques. A fornix-based conjunctival flap was performed, and after hemostasis of the episclera was attained, MMC $(0.4 \mathrm{mg} / \mathrm{ml})$ was applied between the sclera and Tenon's capsule with a Weckcell sponge for 3-5 min (mean, $3.5 \mathrm{~min}$ ) according to the surgeon's estimation for each individual patient. After the entire area was carefully irrigated, a rectangular $5 \times 5 \mathrm{~mm}$ scleral flap of one-third to one-half scleral thickness was dissected. The Ahmed glaucoma valve device (model S2 or model FP7, New World Medical Inc, Rancho Cucamonga, California, USA) was sutured to the sclera $10 \mathrm{~mm}$ posterior to the limbus. The distal tube was cut slightly longer than usual before anterior chamber insertion and was inserted into the anterior chamber through a 23-gauge needle track after the anterior chamber was deepened with viscoelastics (Duovisc, Alcon Laboratories, Inc, Fort Worth, TX). Then, the limbal portion of the tube was fixed with 10-0 nylon suture to the deep scleral bed. The scleral flap was closed with 10-0 nylon sutures followed by conjunctival closure.

The procedure was considered a complete success if the long-term IOP was $\leq 21 \mathrm{mmHg}$ without glaucoma medications or further filtration surgery for the entire course of available follow-up, a qualified success if the IOP $<21$ $\mathrm{mmHg}$ was obtained with adjunctive medications, and a failure if the IOP was $>21 \mathrm{mmHg}$ with medications or when further surgery was required, loss of vision to no light perception (NLP) occurred, or phthisis bulbi developed.

\section{Measurements}

Baseline demographic and clinical information was collected for enrolled patients. Postoperative follow-up visits were scheduled at 1 day, 1 week, 2 weeks, 1 month, 3 months, 6 months, 1 year, and 2 years, and the last available follow-up after the procedure. Each examination included measurement of best corrected visual acuity, IOP, slit-lamp biomicroscopy, and ophthalmoscopy. Use of supplemental medical therapy and any surgical complications were documented at each follow-up visit.

\section{Statistical analysis}

Statistical analyses were performed with SPSS software (version 18.0, SPSS Inc., Chicago, IL). We used paired, two-tailed Student's $t$-tests to compare preoperative and postoperative data; an unpaired, two-tailed Student's $t$-test for continuous variables; Wilcoxon signed rank tests for nonparametric variables; and chi-square tests for comparison of the success rates between two subgroups. Kaplan-Meier survival curves were computed for all patients and two subgroups. Probability values of $<0.05$ were considered significant.

\section{Results}

\section{Baseline characteristics}

The clinical characteristics of the 18 patients are summarized in Table 1 , including 4 male patients and 14 female 
Table 1 Summary of the baseline characteristics of 18 patients with ICE syndrome.

\begin{tabular}{|c|c|c|c|c|c|c|c|c|}
\hline Patient number & Age (yrs) & Gender & $\begin{array}{l}\text { Eye } \\
\text { (OD or OS) }\end{array}$ & Diagnosis & $\begin{array}{l}\text { Previous } \\
\text { surgery }\end{array}$ & $\begin{array}{l}\text { Preoperative } \\
\text { IOP }(\mathrm{mmHg})\end{array}$ & $\begin{array}{l}\text { Preoperative } \\
\text { visual acuity }\end{array}$ & $\begin{array}{l}\text { No. of preoperative } \\
\text { medications }\end{array}$ \\
\hline 1 & 47 & M & OS & Iris atrophy & $\begin{array}{l}\text { Trab } \\
\text { with MMC }\end{array}$ & 48 & $20 / 25$ & 4 \\
\hline 2 & 46 & $\mathrm{~F}$ & OS & Chandler & $\begin{array}{l}\text { Trab } \\
\text { with MMC } \times 2\end{array}$ & 36 & $20 / 25$ & 4 \\
\hline 3 & 34 & $\mathrm{~F}$ & OS & Chandler & $\begin{array}{l}\text { Trab } \\
\text { with MMC }\end{array}$ & 37 & $20 / 60$ & 4 \\
\hline 4 & 33 & $\mathrm{~F}$ & OD & Chandler & Trab & 25 & $\mathrm{CF}$ & 3 \\
\hline 5 & 54 & $\mathrm{~F}$ & OD & Iris atrophy & $\begin{array}{l}\text { Trab, Trab } \\
\text { with MMC }\end{array}$ & 43 & $20 / 40$ & 4 \\
\hline 6 & 33 & $\mathrm{~F}$ & OD & Iris nevus & Trab & 29 & $20 / 60$ & 3 \\
\hline 7 & 42 & M & OD & Iris nevus & None & 30 & $20 / 30$ & 4 \\
\hline 8 & 37 & $\mathrm{~F}$ & OS & Chandler & Trab & 42 & $20 / 30$ & 4 \\
\hline 9 & 54 & $\mathrm{~F}$ & OS & Iris atrophy & None & 21 & $20 / 400$ & 3 \\
\hline 10 & 25 & $\mathrm{~F}$ & OS & Iris nevus & None & 21 & $\mathrm{CF}$ & 3 \\
\hline 11 & 33 & M & OS & Chandler & None & 35 & $20 / 20$ & 3 \\
\hline 12 & 36 & $\mathrm{~F}$ & OD & Iris nevus & None & 35 & $20 / 50$ & 4 \\
\hline 13 & 31 & $\mathrm{~F}$ & OD & Iris nevus & $\begin{array}{l}\text { Trab } \\
\text { with MMC }\end{array}$ & 36 & $20 / 60$ & 3 \\
\hline 14 & 34 & $\mathrm{~F}$ & OD & Iris atrophy & None & 31 & $20 / 200$ & 3 \\
\hline 15 & 49 & $\mathrm{~F}$ & OS & Chandler & Trab & 43 & $20 / 50$ & 3 \\
\hline 16 & 38 & $\mathrm{~F}$ & OD & Iris atrophy & None & 44 & $\mathrm{CF}$ & 4 \\
\hline 17 & 42 & $\mathrm{~F}$ & OD & Iris atrophy & $\begin{array}{l}\text { Trab } \\
\text { with MMC }\end{array}$ & 24 & $20 / 25$ & 4 \\
\hline 18 & 52 & M & OD & Chandler & None & 56 & NLP & 4 \\
\hline
\end{tabular}

Iris atrophy essential iris atrophy, Chandler Chandler syndrome, Iris nevus Iris nevus syndrome, Trab trabeculectomy, $M M C$ mitomycin-c, $C F$ count fingers, $N L P$ no light perception

patients. The mean age, mean preoperative IOP, and median number of preoperative glaucoma medications were $40 \pm$ 8.6 (25-54) years, $34.8 \pm 10.6(12-56) \mathrm{mmHg}$, and $3.6 \pm$ 0.5 medications, respectively. Baseline visual acuity ranged from 20/20 to NLP. All eyes were phakic. Seven eyes were diagnosed with Chandler syndrome, six essential iris atrophy, and five Cogan-Reese syndrome. Six eyes received a model S2 Ahmed aqueous shunt, and the remaining 12 eyes received a model FP7 Ahmed shunt. All tubes were implanted in the anterior chamber. The follow-up period ranged from 12 to $77(42.0 \pm 19.3)$ months.

There were no significant differences between the Trab group and the no-Trab group concerning age, baseline IOP, number of preoperative glaucomatous medications and follow-up period $(P$ values all $>0.1)$.

\section{Postoperative results}

The outcomes of all the subjects are shown in Table 2. The mean IOP was $17.9 \pm 3.7 \mathrm{mmHg}(t=7.124, P=0.000)$ at 1 year and $16.9 \pm 5.2 \mathrm{mmHg}(t=6.149, P=0.000)$ at 2 years. At the last follow-up visit, the mean IOP was significantly decreased to $17.4 \pm 4.9 \mathrm{mmHg}(8-28 \mathrm{mmHg})$ compared with the baseline IOP $(t=6.791, P=0.000)$, and the number of glaucoma medications decreased to $1.6 \pm 1.1(Z$ $=-3.545, \quad P=0.000)$. Of the 18 eyes, five $(27.8 \%)$ achieved complete success, nine $(50.00 \%)$ were qualified successes, and the remaining four $(22.2 \%)$ were considered failures. Figure 1 shows the Kaplan-Meier survival curves for all eyes being studied. The average survival time was $64 \pm 6$ months (median, 47 months). Survival was $94.4 \%$ at 1 year, $88.1 \%$ at 2 years, and $73.5 \%$ at 3 years.

Three patients in the no-Trab group maintained adequate IOP control without medications $(3 / 8,37.5 \%)$, and two patients in the Trab group achieved complete success $(2 / 10$, $20 \%$ ). Two cases in each subgroup failed based on uncontrolled IOP with glaucoma medications following aqueous shunt implant surgery. A chi-square test was performed, but no significant difference was found when we compared the complete success rate or the overall success rate between the two subgroups $\left(\chi^{2}=0.678, P=0.410\right.$, and $\chi^{2}=0.064$, $P=0.800$, respectively). Figure 2 shows the Kaplan-Meier survival curves for the two subgroups. Kaplan-Meier survival analysis with the log-rank test showed no differences 


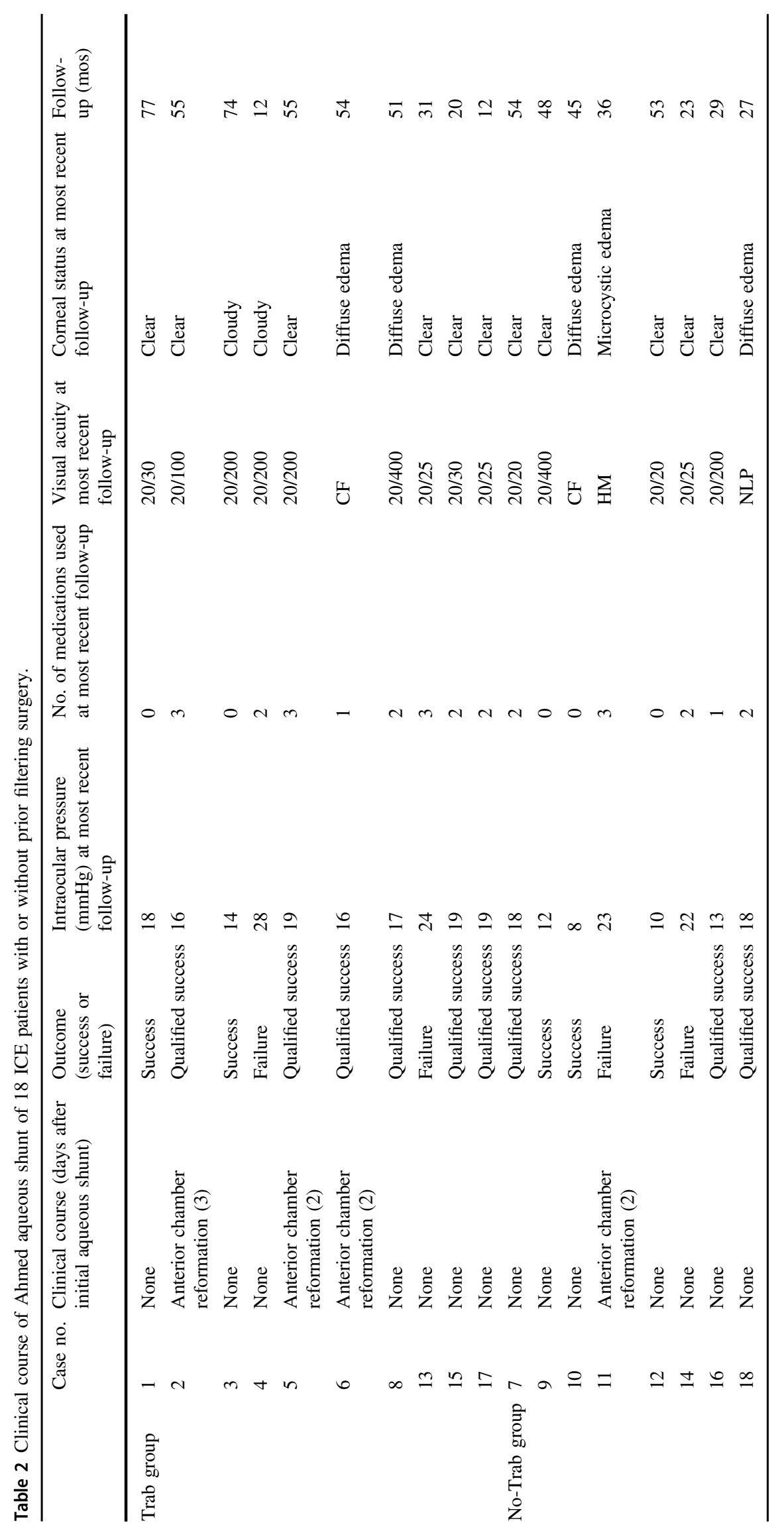


Fig. 1 Kaplan-Meier survival curves for all patients. The line plots the cumulative probabilities against time that the intraocular pressure (IOP) remains below $21 \mathrm{mmHg}$ with or without additional medical treatment (complete success and qualified success).

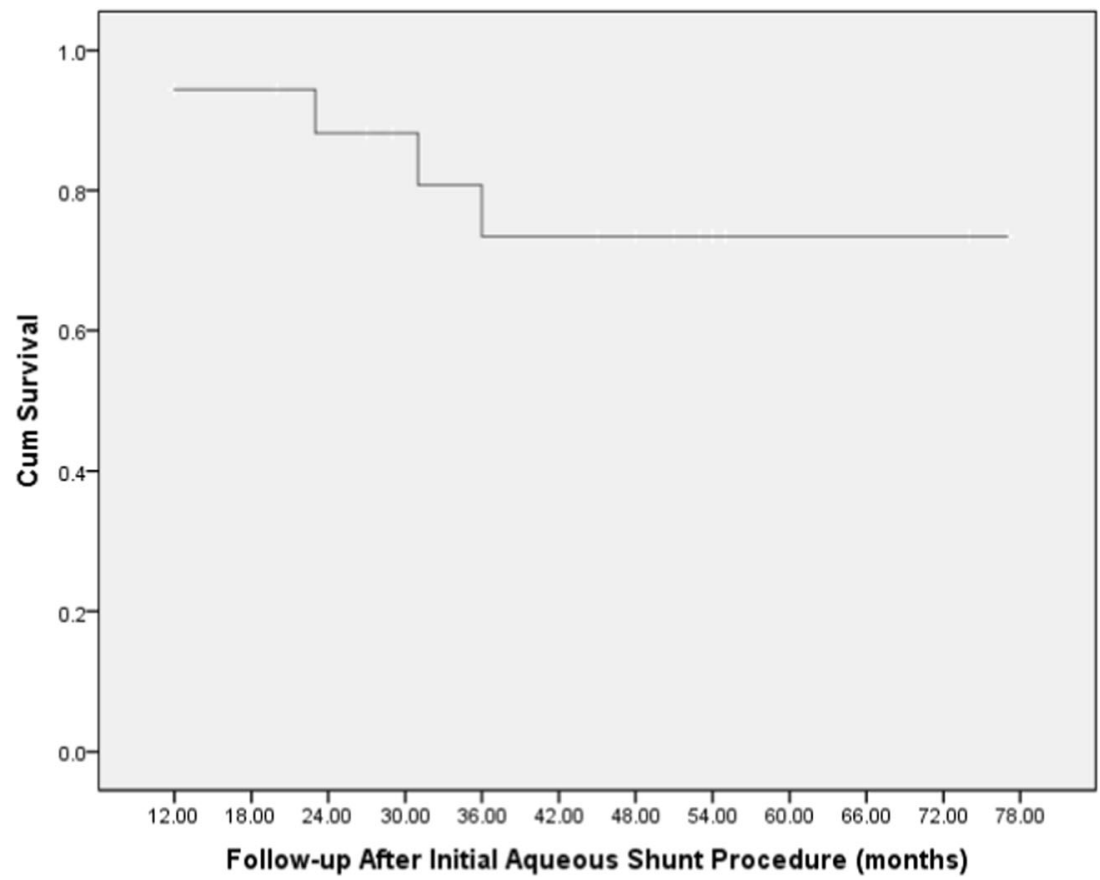

Fig. 2 Kaplan-Meier survival curves for the Trab group and no-Trab group. The curves show the cumulative probabilities against time that the intraocular pressure (IOP) remains below $21 \mathrm{mmHg}$ with or without additional medical treatment (complete success and qualified success). The solid line represents the Trab group, and the dotted line the no-Trab group. Comparison using the log-rank test showed no differences in survival between the Trab group and the no-Trab group $\left(\chi^{2}=0.064, P=0.800\right)$.

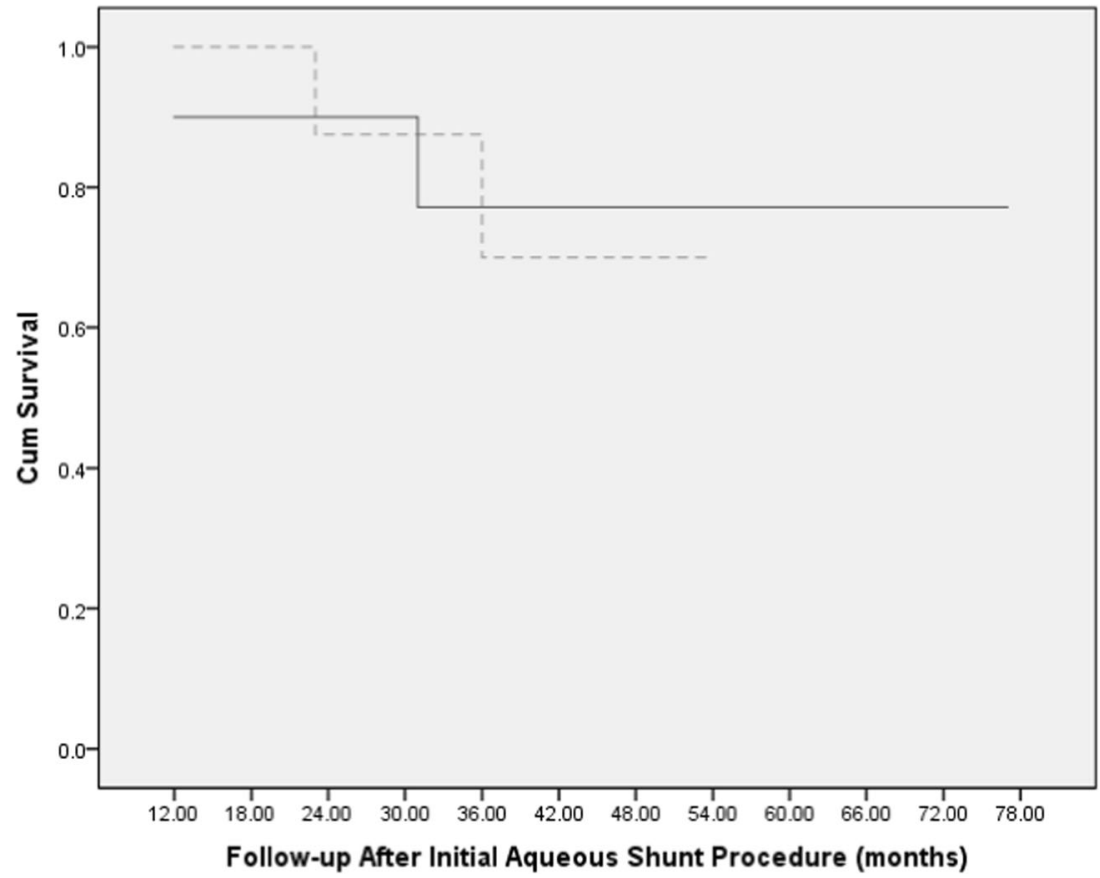

in survival between the Trab group and the no-Trab group $\left(\chi^{2}=0.064, P=0.800\right)$.

The postoperative visual acuity ranged from NLP to 20/ 20 , eight eyes had improved visual acuity compared with the baseline, three remained unchanged, and seven had worse visual acuity. In these seven cases, one (case 3) had cloudy cornea, two (case 6,8) had diffuse corneal edema, and one (case 11) had a completely decompensated cornea with microcystic edema. Cases 3 and 8 had shown mild corneal edema prior to aqueous shunt implantation, and although case 3 achieved success and case 8 qualified success, decrease in vision due to progressive corneal decompensation happened at postoperative 60 months and 24 months respectively. Both case 6 and case 11 suffered from a postoperative flat anterior chamber and received anterior chamber reformation. Case 6 achieved qualified success but had diffuse corneal edema at 19 months postoperatively. Case 11 had an IOP of 23 
mmHg while taking multiple glaucoma medications and had corneal decompensation at 8 months after tube implantation. Eyes with improved visual acuity all maintained relatively clear corneas during follow-up.

Four cases (cases 2, 5, 6, and 11) in our study displayed hypotony and a flat anterior chamber in the first $2-5$ postoperative days, among which case 5 developed choroidal detachment confirmed by ultrasonic-B scanning examination. The Seidel test was performed in each patient and no conjunctival wound leakage was identified. To avoid possible damage to the compromised corneal endothelium, we performed anterior chamber reformation immediately once the conservative treatment failed to deepen the anterior chamber within 1-2 days. Balanced salt solution was injected into the anterior chamber through the previous corneal paracentesis site and no conjunctival leakage was further confirmed. Then the viscoelastic material was injected at the same site until the anterior chamber became deep. No shallow anterior chamber recurred after a single surgical intervention. On the last follow-up visit, cases 2, 5, and 6 had achieved qualified success. No other complications related to glaucoma drainage implants, such as tube blockade by iris, tube exposure, tube reposition, encapsulated bleb, diplopia, suprachoroidal hemorrhage or endophthalmitis, occurred during our study.

\section{Discussion}

Patients with ICE syndrome have been reported to have a higher failure rate after filtration surgery than most types of glaucoma [5, 7-10]. The relatively young age of onset and the progressive nature of ICE syndrome with membrane proliferation and formation of anterior synechiae predispose the patient to excessive fibrosis and obstruction of the ostium of the filtration site. Kidd et al. [7] reported a $64 \%$ survival at 1 year and only $39 \%$ at 3 years after trabeculectomy in a series of 37 patients. Twenty-four of these trabeculectomy patients required a second surgery, and eight required a third surgery.

Antifibroblastic adjuncts such as MMC and 5fluorouracil (5-FU) may improve the success rate of trabeculectomy by inhibiting fibroblast proliferation at the filtering site. However, since this is not the primary mechanism of failure in eyes with ICE syndrome, trabeculectomy with adjunctive antifibrotic agents was also described as relatively ineffective in ICE patients [10-12]. Trabeculectomy with postoperative subconjunctival injections of 5-FU was examined by Wright et al. [12]. In a group of nine patients, sufficient IOP control was achieved in only four eyes after 6-54 months of follow-up. Doe et al. [8] in a series of 12 patients reported a success rate of $73 \%$ at 1 year, $44 \%$ at 3 years, and $29 \%$ at 5 years after antifibrotic augmented trabeculectomy. In their study, 5-FU was used in nine eyes and MMC in three eyes.

Glaucoma drainage devices (GDDs) may create an alternate aqueous pathway from the anterior chamber by channeling aqueous out of the eye through a tube to a subconjunctival bleb or to the suprachoroidal space, thus bypassing the chief hazard of filtration procedures caused by the regrowth of an ICE membrane over the ostium for filtration and appearing to result in a higher success rate compared with trabeculectomy in ICE patients [13].

In the current series, we followed 18 ICE patients who underwent Ahmed aqueous shunt surgery with adjunctive MMC. Five eyes (27.8\%) achieved adequate IOP control without glaucoma medications or further surgeries, nine $(50.00 \%)$ were qualified successes and the remaining four (22.2\%) were considered failures. The success rate is comparable to previous results reported by Kim et al. and Doe et al. Kim et al. [9] reported a case series of ten ICE patients undergoing aqueous tube shunt surgery in 1999, and the implant used was a Molteno double-plate in six eyes, a Schocket implant in three eyes, and a Baerveldt shunt in one eye. After a median follow-up of 55 months, seven eyes $(70 \%)$ had adequate IOP control. Doe et al. [8] also used various types of GDDs in their 21 cases, including 13 Baerveldt shunts, 7 Molteno shunts and one Ahmed shunt. Of the 21 eyes, $14(66 \%)$ maintained good vision and adequate control of IOP.

Ten patients (Trab group) in our study underwent trabeculectomy prior to aqueous shunt surgery. The results showed that there were no significant differences in the complete success rate, overall success rate, or survival rate between the Trab group and the no-Trab group. Since the success rate of filtration surgery declines with each subsequent procedure and aqueous shunt implantation appears to result in a higher success rate in ICE patients than filtration surgery, early consideration should be given to a GDD procedure in patients in whom trabeculectomy fails.

In Kim et al. [9] series, the main reason for aqueous shunt surgery failure was blockage of the tube ostium by the iris, ICE membrane, or membrane-induced tube migration, and five of the ten patients required one or more tube repositioning or revisions. Doe et al. [8] also found tube ostium blockage by the iris and/or ICE membrane or anterior migration of the tube secondary to the ICE membrane in four cases, which required repositioning or replacement of the distal tube. It was noticed that tube blocking by the iris and/or tube repositioning did not occur in our study. The lower rate of tube dislocation may either reflect good initial positioning of the tube or less aggressive endothelial migration. The optimal positioning of a tube in a phakic eye, especially one with an anteriorly displaced iris due to contraction of an ICE membrane, can be challenging. 


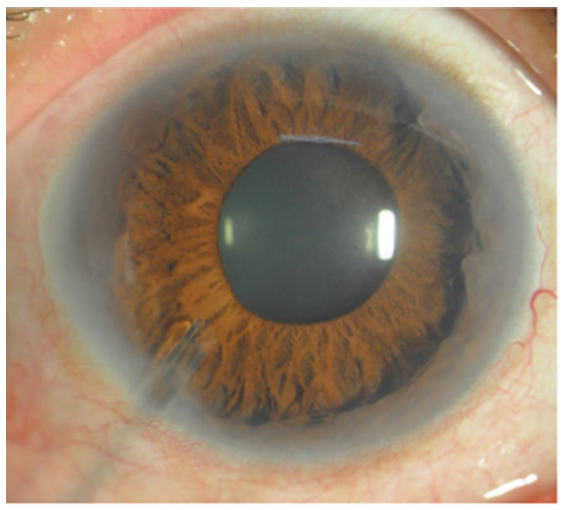

Fig. 3 Slit-lamp photographs of the aqueous shunt implant in the right eye of an ICE patient. The patient had broad peripheral anterior synechiae in the right eye with only 12 O'clock and the temperoinferior quadrant uninvolved. Thus, the Ahmed aqueous tube was inserted in the temperoinferior quadrant with the least ICE membrane

To minimize the risk of tube occlusion or anterior migration, surgical techniques of aqueous shunt implantation can be modified as follows: (1) choose the optimal tube insertion site without or with the least ICE membrane and PAS, but not the conventional site in the nasosuperior or temperosuperior quadrant; (2) insert the tube approximately $0.5 \mathrm{~mm}$ from the limbus through a 23 -gauge needle track under the scleral flap and fix the limbal portion of the tube with 10-0 nylon suture to the deep scleral bed. A small size needle entry, a proper length scleral tunnel and a good fixation suture will be beneficial for avoiding dislocation of the tube; (3) use viscoelastics to separate the iris synechiae and deepen the anterior chamber before tube insertion to avoid tube ostium blocking by the iris; (4) cut the distal tube slightly longer than usual before anterior chamber insertion and keep the tube tip away from potential sources of ICE cells such as the iris and the cornea and away from the iris anterior synechiae (Fig. 3).

Tube insertion through the ciliary sulcus has been reported to help to avoid damage to cornea by preventing the tube from contacting corneal endothelium in eyes with a posterior chamber intraocular lens [14-16]. The anteriorposterior length of the sulcus will be increased after cataract extraction, due to the smaller thickness of the implant compared with the crystalline lens. Therefore, inserting tubes through the ciliary sulcus would be preferred in pseudophakic ICE eyes. In our series, all patients were phakic. To avoid possible damage to the compromised corneal endothelium caused by postoperative shallow anterior chamber and tube-corneal touch, we chose the Ahmed implant, the only commercially available flowrestricted glaucoma drainage implant, for all patients in our series. Although the flow restrictor of the Ahmed valve provides an added level of safety by limiting aqueous humor flow when the IOP becomes too low, the main

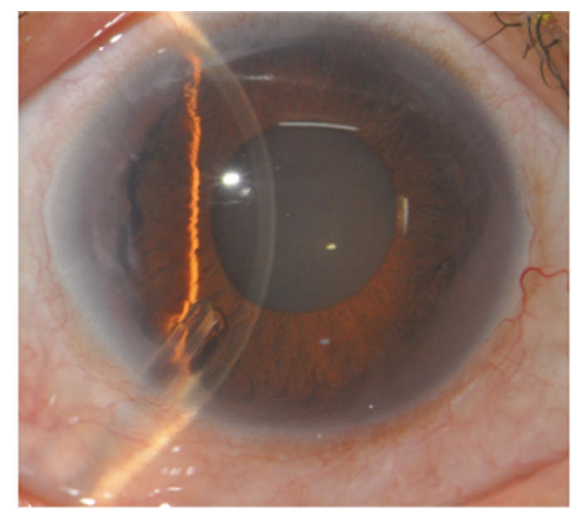

to minimize the risk of tube occlusion, but not the conventional site in the nasosuperior or temperosuperior quadrant. In addition, the distal tube was cut slightly longer than usual to keep the tube tip away from potential sources of ICE cells.

complication in our series was still hypotony and a shallow anterior chamber. The reported rate of postoperative shallow anterior chamber has been variable in different Ahmed valve studies. The Ahmed Baerveldt Comparison Study stated a rate of $19 \%$ [17], but Choo's study reported a value of only $2.63 \%$ [18]. Intraoperatively injecting the appropriate volume of viscoelastics to deepen the anterior chamber is crucial to reduce the rate of postoperative flat anterior chamber $[19,20]$. The anterior chamber did not exhibit recurrence of shallowing in these four cases after a single surgical intervention. Since this complication was transient and showed a good response to the treatment, and because no other complications related to glaucoma drainage implants occurred during our study, our experience indicated that Ahmed glaucoma valve implantation is a safe technique in decreasing the IOP in eyes with ICE syndrome.

In summary, with modified surgical procedures, Ahmed aqueous shunt surgery appears to be an effective method for IOP lowering in glaucoma secondary to ICE syndrome when medical treatment or conventional filtration surgeries fail. Early consideration may be given to aqueous shunt surgery in these patients when trabeculectomy fails. Since this is a retrospective study with a small sample size, a large, randomized, prospective study would be necessary to prove whether aqueous shunt surgery would be the first choice in patients with glaucoma secondary to ICE syndrome.

\section{Summary}

\section{What was known before}

- Glaucoma secondary to ICE syndrome is refractory and the success rate of conventional filtering surgery is poor 
in the long term. Aqueous shunt implants have been proven to be effective in treating glaucoma in ICE syndrome, but there was high rate of the blocking of the tube ostium by iris, ICE membrane, or membraneinduced tube migration, which was the main reason for surgery failure.

\section{What this study adds}

- With modified surgical procedures, the risk of tube occlusion or anterior migration is minimized. The modified surgical techniques include: (1) choose the optimal tube insertion site (2) a good fixation suture (3) use viscoelastics to separate the iris synechiae and deepen the anterior chamber before the tube insertion (4) cut the distal tube slightly longer than usual.

\section{Compliance with ethical standards}

Conflict of interest The authors declare that they have no conflict of interest.

Publisher's note Springer Nature remains neutral with regard to jurisdictional claims in published maps and institutional affiliations.

\section{References}

1. Silva L, Najafi A, Suwan Y, Teekhasaenee C, Ritch R. The iridocorneal endothelial syndrome. Surv Ophthalmol. 2018;63:665-76.

2. Levy SG, McCartney AC, Baghai MH, Barrett MC, Moss J. Pathology of the iridocorneal-endothelial syndrome. The ICE-cell. Investig Ophthalmol Vis Sci. 1995;36:2592-601.

3. Bromley JG, Randleman JB, Stone D, Stulting RD, Grossniklaus HE. Clinicopathologic findings in iridocorneal endothelial syndrome and posterior polymorphous membranous dystrophy after Descemet stripping automated endothelial keratoplasty. Cornea. 2012;31:1060-4.

4. Liu YK, Wang IJ, Hu FR, Hung PT, Chang HW. Clinical and specular microscopic manifestations of iridocorneal endothelial syndrome. Jpn J Ophthalmol. 2001;45:281-7.
5. Laganowski HC, Kerr Muir MG, Hitchings RA. Glaucoma and the iridocorneal endothelial syndrome. Arch Ophthalmol. 1992;110:346-50.

6. Walkden A, Au L. Iridocorneal endothelial syndrome: clinical perspectives. Clin Ophthalmol. 2018;12:657-64.

7. Kidd M, Hetherington J, Magee S. Surgical results in iridocorneal endothelial syndrome. Arch Ophthalmol. 1988;106:199-201.

8. Doe EA, Budenz DL, Gedde SJ, Imami NR. Long-term surgical outcomes of patients with glaucoma secondary to the iridocorneal endothelial syndrome. Ophthalmology. 2001;108:1789-95.

9. Kim DK, Aslanides IM, Schmidt CM Jr, Spaeth GL, Wilson RP, Augsburger JJ. Long-term outcome of aqueous shunt surgery in ten patients with iridocorneal endothelial syndrome. Ophthalmology. 1999;106:1030-4.

10. Chandran P, Rao HL, Mandal AK, Choudhari NS, Garudadri CS, Senthil S. Outcomes of primary trabeculectomy with mitomycin-c in glaucoma secondary to iridocorneal endothelial syndrome. $\mathrm{J}$ Glaucoma. 2016;25:e652-6.

11. Lanzl IM, Wilson RP, Dudley D, Augsburger JJ, Aslanides IM, Spaeth GL. Outcome of trabeculectomy with mitomycin-C in the iridocorneal endothelial syndrome. Ophthalmology. 2000; 107:295-7.

12. Wright MM, Grajewski AL, Cristol SM, Parrish RK. 5Fluorouracil after trabeculectomy and the iridocorneal endothelial syndrome. Ophthalmology. 1991;98:314-6.

13. Imamoglu S, Sevim MS, Yıldız HE, Vural ET, Bardak H, Bardak Y. Surgical outcomes of patients with iridocorneal endothelial syndrome: a case series. Int Ophthalmol. 2017;37:607-13.

14. Weiner A, Cohn AD, Balasubramaniam M, Weiner AJ. Glaucoma tube shunt implantation through the ciliary sulcus in pseudophakic eyes with high risk of corneal decompensation. J Glaucoma. 2010;19:405-11.

15. Prata TS, Mehta A, De Moraes CG, Tello C, Liebmann J, Ritch R. Baerveldt glaucoma implant in the ciliary sulcus: midterm followup. J Glaucoma. 2010;19:15-18.

16. Bayer A, Onol M. Clinical outcomes of Ahmed glaucoma valve in anterior chamber versus ciliary sulcus. Eye. 2017;31:608-14.

17. Budenz DL, Barton K, Feuer WJ, Schiffman J, Costa VP, Godfrey DG, et al. Treatment outcomes in the Ahmed Baerveldt Comparison Study after 1 year of follow-up. Ophthalmology. 2011;118:443-52.

18. Choo JQH, Chen ZD, Koh V, Liang S, Aquino CM, Sng C, et al. Outcomes and complications of Ahmed tube implantation in Asian eyes. J Glaucoma 2018;27:733-8.

19. Mokbel TH, Khalaf MA, El-Khouly SE, El-Metwally NO. Flexible Ahmed valve for selected cases of refractory glaucoma. Eur J Ophthalmol. 2012;22:83-9.

20. Gedde SJ, Panarelli JF, Banitt MR, Lee RK. Evidenced-based comparison of aqueous shunts. Curr Opin Ophthalmol. 2013; 24:87-95. 